\title{
Relevant Applications of Scanning Electron Microscopy in a Pharmaceutical Development Laboratory
}

\author{
Ronald L. Mueller*
}

*GlaxoSmithKline, Worldwide Physical Properties-Pennsylvania, King of Prussia, PA 19406

This presentation provides examples where conventional scanning electron microscopy (SEM) contributed significantly in the drug development process. It also presents a general overview of how the SEM, in conjunction with other various analytical techniques, functions in a physical characterization group within the Pharmaceutical Industry. When SEM is used in a multidisciplinary approach, it becomes a powerful characterization tool for the development of pharmaceutical materials.

Three case studies are presented. Firstly, an efficient mixing process for a drug substance with various excipients was required.[1] However, the drug substance was an oncological agent and red iron oxide was used as a substitute since it could be easily handled during the processing investigations, had a similar particle size distribution and tended to agglomerate during the mixing process. Under the same processing conditions in a high shear mixer, calcium phosphate dibasic excipients were evaluated for their mixing efficiency with $1 \% \mathrm{w} / \mathrm{w}$ red iron oxide. The mixing efficiency was followed by colorimetry and was collaborated by using energy dispersive x-ray (EDX) spectroscopy. Figure 1 displays EDX overlay maps for phosphorous and iron at two different time points during the processing investigation of A-Tab. Two of the three calcium phosphate dibasic excipients investigated mixed well.

Secondly, a laser light diffraction method was modified so that it could discriminate between "as is" and micronized materials. The laser light diffraction data was complimented by the image analysis results obtained by optical microscopy. Figure 2 displays the "as is" and micronized materials used in this study.

Thirdly, by using EDX spectroscopy, it was demonstrated why some core tablets manufactured from newer drug substance batches failed content uniformity. Whereas core tablets produced from older drug substance batches or reworked newer drug substance batches passed content uniformity. Figure 3 displays EDX overlay maps for carbon and sulfur for core tablets manufactured from a newer drug substance batch which failed and then passed content uniformity after reworking the core tablets.

\section{References}

[1] R. Abramowitz et al., "Effect of Carrier on the Mixing Efficiency of a Cohesive Powder: Red Iron Oxide," presented at the 1999 AAPS Annual Meeting and Exposition. 

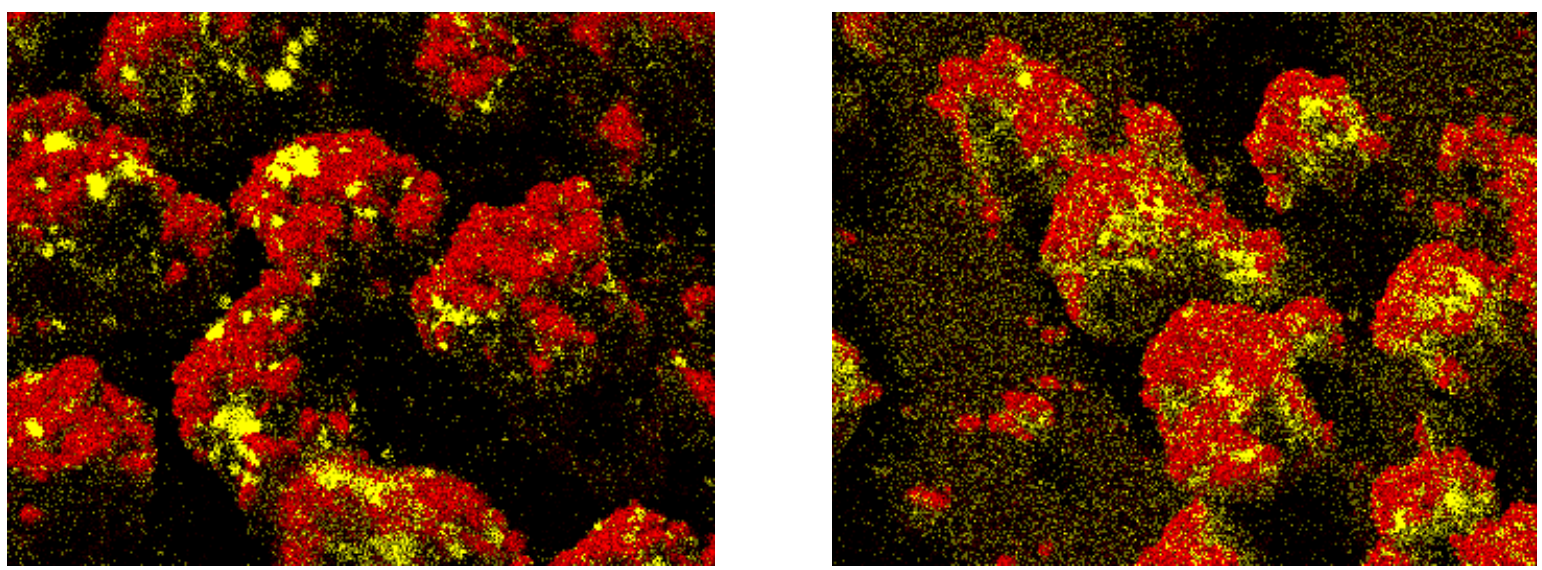

Figure 1. EDX overlay maps at 200X for phosphorous (red) and iron (yellow) at 15 seconds (left image) and 5 minutes (right image) during the processing investigation of "A-Tab."
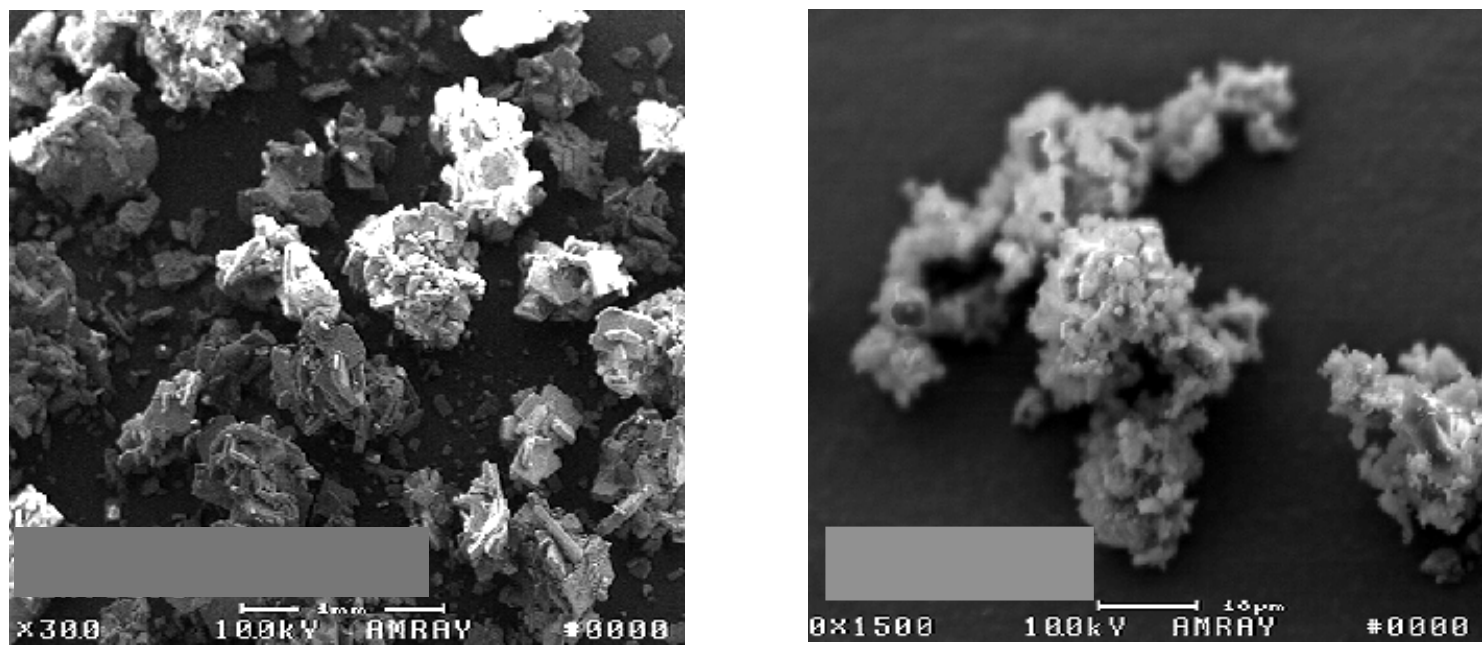

Figure 2. SEM images of "as is" (left image) and micronized (right image) materials.
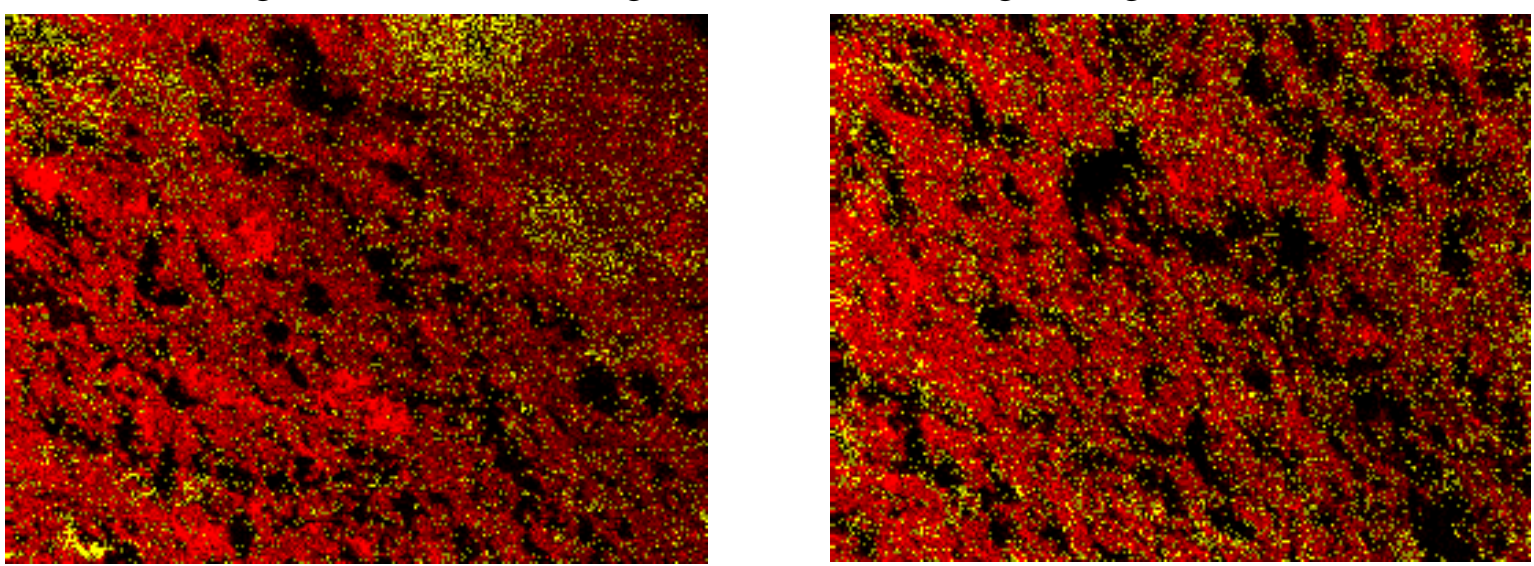

Figure 3. EDX overlay maps for carbon (red) and sulfur (yellow) for core tablets manufactured from a newer drug substance batch which failed (left image) and then passed (right image) content uniformity after reworking the core tablets. 